\title{
Quantitative histopathology identifies patients with thin melanomas who are at risk for
}

\section{metastases}

Running head: Metastatic thin melanomas

Evan S. Glazer, MD, PhD $;{ }^{1}$ Peter H. Bartels, PhD; ${ }^{2}$ Fangru Lian, MD $;{ }^{1}$ Stephanie T. Kha; ${ }^{3}$ Sherif S.

Morgan, PhD; ${ }^{2}$ Vinicius D. da Silva, MD; ${ }^{4}$ Michael L. Yozwiak, MS; ${ }^{2}$ Hubert G. Bartels, MSEE $;{ }^{2}$ Lee

D. Cranmer, MD, $\mathrm{PhD} ;{ }^{2}$ Jefferson K. de Oliveira, $\mathrm{MD} ;{ }^{4}$ David S. Alberts, $\mathrm{MD} ;{ }^{2}$ James A. Warneke, $\mathrm{MD} ;{ }^{1}$ Robert S. Krouse, $\mathrm{MD}^{1,5}$

${ }^{1}$ University of Arizona College of Medicine

${ }^{2}$ University of Arizona Cancer Center

${ }^{3}$ University of Arizona College of Science

${ }^{4}$ Hospital Sao Lucas, Pontificia Universidade Católica do Rio Grande do Sul, Porto Alegre, Brazil

${ }^{5}$ Southern Arizona Veterans Affairs Health Care System

Corresponding author

Robert S, Krouse, MD. FACS

Professor of Surgery

Southern Arizona VA Health Care System

Surgical Care line, 2-112

3601 S. 6th Ave

Tucson AZ 85723

(520) $792-1450$

robert.krouse@,va.gov

Conflict of interest: Two authors (PHB and DSA) own patents related to premalignant lesions and 
karyometry. The other authors declare that they have no conflicts of interest.

Previous dissemination: Portions of this research were presented at the Academic Surgical Congress, New Orleans, February 2013 


\begin{abstract}
Objective: This small exploratory study was designed to test the hypothesis that thin melanoma lesions contain nuclei of two similar phenotypes, in different proportions. In lesions likely to progress to metastatic disease one of these phenotypes predominates.
\end{abstract}

Methods: Histopathological sections from 18 cases of thin melanomas which did not progress to metastasis, and from 10 cases which did so were imaged and digitized at high resolution, with a total of 2,084 and 1,148 nuclei, respectively, recorded. Five karyometric features were used to discriminate between nuclei from indolent and from potentially metastatic lesions. For each case, the percentage of nuclei classified by the discriminant function as having come from a potentially metastatic lesion was determined and termed case classification criterion.

Results: Standard histopathological criteria, such as ulceration and high mitotic index, indicated in this material the need for intensive therapy for only one of the ten participants, as compared to $7 / 10$ identified correctly by the karyometric measure. Using as a case classification criterion threshold of $40 \%$, the overall accuracy was $86 \%$ in the test set.

Conclusions: The proportion of nuclei of an aggressive phenotype may lend itself as an effective prognostic clue for thin melanoma lesions. The algorithm developed in this training set appears to identify those patients at high risk for metastatic disease, and demonstrates a basis for a further study to assess the utility of prognostic clues for thin melanomas.

Key words: aggressive melanoma, thin melanoma, karyometry, quantitative histopathology 


\section{Introduction}

The present study was conducted as an exploratory probe to test the hypothesis that thin malignant melanoma lesions comprise two phenotypes of nuclei and that lesions which have the potential to progress to metastatic disease contain a higher proportion of one of these two phenotypes of nuclei. The hypothesis was prompted by results obtained from recent studies of clinical materials from atypical hyperplasia of the endometrium (AEH) [1] and from cutaneous squamous cell cancer $(\mathrm{cSCC})[2,3]$.

For both lesions of AEH and cSCC, there are two axes of dispersion for karyometric variables. One axis involves variables known to undergo a monotonic increase in value with progression to malignant disease, such as total optical density and relative nuclear area. The other, independent axis of dispersion separates cases with potential to progressive development from cases that remain indolent. The distinguishing karyometric features here reflect the spatial and statistical distribution of the nuclear chromatin. Changes in the chromatin distribution pattern are not necessarily visually perceived, but have been established and statistically secured by image analytic procedures. In the study of cSCC lesions up to $85 \%$ of cases with poor prognosis could be recognized on that basis. For both of the above-mentioned lesions, the following quantities were established: the presence of two phenotypes of nuclei, and the association of a high proportion of nuclei of one of these phenotypes with invasive disease or aggressive disease development.

Melanomas that are $0.75 \mathrm{~mm}$ to $1 \mathrm{~mm}$ thick have approximately $5 \%$ risk of metastasis; those less than $0.75 \mathrm{~mm}$ thick metastasize even less frequently [4-6]. Patients with melanomas thicker than 1 mm have a higher risk of metastasis, and they often undergo sentinel lymph node biopsy because of the thickness of their melanomas. Currently the most powerful prognostic features in patients with thin cutaneous melanomas ( $<1 \mathrm{~mm}$ thick) are tumor depth/thickness, ulceration [4], and mitosis [5]. Usually, the initial site of metastasis is the regional lymph node basis [7]. Given the low probability of 
metastasis in patients with thin melanomas, identification of those melanomas that are at highest risk represents an opportunity for more personalized surgical treatment. However, as Bjoernhagen [8] has emphatically pointed out, even thin melanomas may metastasize and the above criteria may not unequivocally predict clinical outcome.

There is a sizable literature dedicated to relating specific prognostic characteristics to thin melanomas. Tan and Baak in 1984 investigated the utility of karyomorphometric characteristics such as nuclear shape for prognosis $[9,10]$. Li et al., though, in 2004 discounted the general efficacy of morphometric criteria, such as nuclear shape factors [11], in the context of their own comprehensive evaluation of potentially useful prognostic clues. A number of studies in malignant melanoma lesions have involved DNA measurements to obtain prognostic indicators [8-11].

The present study is based on a multivariate discriminant analysis of the spatial and statistical distribution of the nuclear chromatin. These procedures have been found to provide an exquisite sensitivity for the detection of small differences between two nuclear phenotypes.

\section{Materials and Methods}

\section{Patients and clinical materials}

The study was approved by the University of Arizona institutional review board. Twenty-eight patients with thin melanomas who underwent resection from January 2006 through December 2008 and who had no evidence of metastasis at diagnosis were retrospectively identified. The index procedures occurred at two institutions, the Arizona Cancer Center $(n=22)$ and Hospital Sao Paulo, Pontifícia Universidade Católica do Rio Grande do Sul $(\mathrm{n}=6)$. Clinicopathologic and follow-up data were reviewed for ulceration and mitotic index.

\section{Patient characteristics}

All 28 patients underwent wide local resection with at least $1 \mathrm{~cm}$ margins. Of the 18 patients in 
the non-metastatic group, 4 had undergone sentinel or auxiliary lymph node biopsy at the discretion of the surgeon at the time of initial excision, as compared to 7 of the 10 patients in the metastatic group. By definition none of the patients in the non-metastatic group were found to have positive lymph nodes during their index procedure, as compared with 3 patients in the metastatic group. We do not precisely know how surgeons decided how to proceed with sentinel node biopsy but factors such as elevated mitotic rate or melanoma thickness close to $1.0 \mathrm{~mm}$ may have caused surgeons to perform sentinel lymph node biopsy.

\section{Clinical outcomes}

At the time of follow-up all patients in the metastatic group had developed both lymph node and distant metastases, as compared to none in the non-metastatic group. The sites of distant metastases included the small intestines, lungs, bones, chest wall, extremities and abdominal wall.

\section{Tumor characteristics}

The thickness of the primary index tumor in the non-metastatic group was $0.53 \pm 0.25 \mathrm{~mm}$; in the metastatic group it was $0.68 \pm 0.26 \mathrm{~mm}(\mathrm{p}=0.20)$. Tumors arose in similar anatomic locations in both groups. None of the melanomas in either group had ulceration. None of the melanomas in the non-metastatic group had a high mitotic index; there was one such case in the metastatic group.

\section{Clinical materials}

The pathologic specimens were fixed in 10\% neutral buffered formaldehyde. Paraffinembedded tumor blocks were obtained from the pathologic archives of the two institutions. Fivemicron sections were cut. Staining with H\&E was done under carefully controlled conditions.

\section{Data recording}

Data were recorded on a videomicrophotometer equipped with a 100:1, N.A. 1.40 plan apochromatic oil immersion objective (NIKON). Relay optics adjusted the magnification for a sampling density of 6 pixels per linear micron. A $610 \mathrm{~nm}$ bandpass interference filter was used to 
enhance contrast.

For the 10 cases where stage III or IV metastatic progression had occurred, 1,148 nuclei were recorded. For the 18 cases with no metastatic progression, 2,084 nuclei were recorded. Two data sets were established, for nuclei from cases with no metastatic progression (M), and for nuclei from cases with metastatic progression (Mmeta).

A set of 93 karyometric features was computed. Feature selection for a discriminant analysis was based on a Kruskal Wallis test [12], and the ambiguity function of Genchi and Mori [13,14].

A Bonferroni correction, a value of $\mathrm{p}<0.0001$ was used as threshold. To derive a classification rule for lesions expected to progress to metastasis versus those expected not to do so, a discriminant function was used. This function was then applied to each case, and the proportion of nuclei assigned to the Mmeta category was determined.

It was hypothesized that lesions of both diagnostic categories each contain the same nuclei of two different phenotypes, but in different proportions. Since nuclei of the two phenotypes cannot be visually identified, training of the discriminant function has to be done on the characteristics of nuclei from lesions with, or with no metastatic progression. The computed karyometric mean values of nuclei thus depend on the relative proportions of the two phenotypes in each case. There is a small difference between the karyometric values of nuclei of pure phenotype and the values used in the discriminant algorithm. It is important therefore to distinguish between a "nucleus from a case of metastatic progression" and a "nucleus of the phenotype predominant in cases of metastasis", because the nuclei from a case of metastatic progression would contain both aggressive and non-aggressive phenotypes.

\section{Results}

The study group included 18 patients with non-metastatic disease (13/18 male) and 10 patients (6/10 male) with metastatic disease. The overall median follow-up time was 4.6 years. The average age 
in the non-metastatic group was $62 \pm 15$ years; in the metastatic group it was $50 \pm 16$ years. In both groups most patients were male.

The notable dispersion of mean values for karyometric variables is shown (Fig. 1), for both diagnostic categories, a bivariate plot of the key variables total optical density and relative nuclear area. The total optical density, reflecting ploidy, varied by a factor of two, and relative nuclear area by a factor of $2-3$. The plot shows that the distribution of cases with potential progression and for cases that remain indolent cover the same range, and the plot also shows that progression to metastatic disease occurs independently of total optical density, i.e., even in cases with near diploid DNA content.

The feature selection for a discriminant analysis identified more than 20 karyometric features with statistically significant differences at $\mathrm{p}<0.0001$. Most of these features represent texture differences of the nuclear chromatin. In the following, all feature values are given in arbitrary, relative units.

\section{Training and test sets}

A training set of five cases of the metastatic lesions $\mathrm{M}$ with 591 nuclei, and of nine cases of the non-metastatic lesions Mmeta, with 1,085 nuclei was formed. Table 1 lists five selected features with their mean values and standardized coefficients in the discriminant function. The classification matrix for nuclei is shown in Table 2. The average accuracy was $64.3 \%$.

The algorithm assigned a negative sign to nuclei from the metastatic cases. The score distributions are shown for the training sets of five and nine cases (Fig. 2). The decision rule was next applied to the test set of five cases from the metastatic lesions, with 557 nuclei, and to the remaining nuclei from the nine cases of non-metastatic lesions, with 896 nuclei.

Using as a case classification criterion the percentage of nuclei assigned to the metastatic lesion category, and a 50\% threshold, the classification resulted, for the training set, in the correct assignment of $4 / 5$, or $80 \%$ of the metastatic lesions, and $6 / 9$, or $67 \%$ of the non-metastatic lesions, for an average 
accuracy of $10 / 14$, or $71 \%$.

For the test set the discriminant function assigned $3 / 5$, or $60 \%$ of the metastatic cases correctly, and $8 / 9$ or $89 \%$ of the non-metastatic cases, for an average accuracy of $11 / 14$, or $79 \%$.

If a $40 \%$ assignment of nuclei to the Mmeta category is used as a case classification criterion the training set data result in an average accuracy of $11 / 14$, or $79 \%$ and the test set data as $12 / 14$ or $86 \%$.

Given the large sample size of 2,084 $+1,148=3,232$ nuclei, the full data sets were submitted to the discriminant algorithm, considering that the large number of nuclei, of more than 1,000 even for the smaller data set, would keep the re-substitution error at a very low percentage [15].

Feature selection identified most of the features previously selected for the training set, with small differences in mean values, for the full data set. Table 1 lists the mean values and standardized coefficients for features previously selected for the training set. The average accuracy was $66.12 \%$. Using the $50 \%$ threshold criterion, the correct classification was $76 \%$. With the $40 \%$ criterion, $89 \%$ of lesions were correctly classified.

The classification matrix for nuclei, with its only marginal discrimination, suggests that the nuclear populations in cases of non-metastatic and of metastatic lesions are very similar. One compatible interpretation is that the similarity is due to the presence, in both data sets, of the same two nuclear phenotypes, and that the only difference between the two diagnostic categories would be due to a difference in proportions of these nuclei, as had been found in cases of cutaneous squamous cell cancer where an aggressive phenotype predominates in cases with poor prognosis [2].

To test this hypothesis, the discriminant function derived above was applied to every individual case in order to determine the percentage of nuclei as having an aggressive/metastatic phenotype. Table 3 lists the percentages of nuclei assigned to the metastatic diagnostic category for both the nonmetastatic and the metastatic data sets. A graphic for the distribution of cases with a certain percentage 
of nuclei assigned to the metastatic category is shown (Fig. 3). Even though the sample size is small, the Mmeta (metastatic) lesions appear to have a predominance of aggressive nuclei.

\section{Discussion}

Only a small percentage of patients with thin melanomas may develop metastasis. It is important to target those high risk patients so that more aggressive treatments and follow-up are offered. Additionally, excessive initial therapy may result in needless complications. Wide local excision and lymph node dissection are associated with complication rates of up to $25 \%$ [16-18]. Even sentinel lymph node biopsy is associated with a short-term complication rate of at least 5\% [19]. Clearly, the decision to proceed with intensive therapy in patients with thin melanoma is not trivial, and how best to identify patients most likely to benefit from intensive therapy remains a clinical dilemma.

The results of the current exploratory study support the hypothesis that thin melanoma lesions contain two phenotypes of nuclei, and that one of these phenotypes predominates in proportion in lesions that eventually result in progression to metastasis. These results thus may serve as a basis for a larger study on more generally representative clinical material, aimed at the development of an unequivocal prognostic indicator for thin melanoma lesions. Such a prognostic clue would be distinctly different from clues as offered by ploidy analysis or by a multivariate analysis of a number of histopathological characteristics.

In this study that applied the standard criteria for aggressive thin melanomas - such as ulceration and high mitotic index - only one of the ten patients in the Mmeta group would have been considered for intensive therapy. In contrast, only five karyometric features descriptive of the nuclear chromatin distribution provided a recognition of more than $70 \%$ of patients who might benefit from intensive therapy. The karyometric features relate to subtle changes in the spatial and statistical distribution of the nuclear chromatin, suggesting not only histone modification but also cellular 
aggression [20].

Chromatin distribution pattern and structure vary widely in patients with melanomas, and multiple subpopulations of malignant phenotypes might exist [21]. Our results are compatible with those observations, and may allow a new avenue to investigate epigenetic changes in patients with thin melanomas $[20,22]$. The concept that subpopulations of cells dominate a tumor's biology is well described in the literature for multiple cancers, including melanomas [23-25].

The results obtained in this study support the hypothesis of the risk for progression to metastatic disease associated with a high proportion of nuclei of a progressive phenotype. The study had a follow-up period of nearly five years. Yet, there clearly are limitations to the generalization of the collected results. The patient numbers were small, and there is the problem of selection bias. Most of the patients underwent sentinel node biopsy at the time of the initial procedure. Thus, patient and tumor characteristics may have alerted the surgeon to the risk of an aggressive phenotype. We do not know how surgeons decided how to proceed with sentinel node biopsy. With the small number of patients, the role of high mitotic index and ulceration could not be adequately investigated. A definitive demonstration of the utility of a karyometric prognostic criterion for thin melanomas should involve stratification for ulceration, mitotic index and tumor stage.

The multiplicity and varied nature of histopathological clues investigated for prognostic efficacy, and the limited clinical utility, reflect the substantial diversity and differences in presentation of malignant melanomas. Overall, the observed lesion diversity leaves the identification of unequivocal prognostic criteria for thin melanoma lesions to future research. However, we have demonstrated in this training set that karyometry appears to identify lesions with high proportions of nuclei that may lead to the early identification of metastatic melanoma. In conclusion, karyometry may have a role in decision-making for patients with thin melanoma. Further study is warranted to examine its utility in this setting. 


\section{References}

1. Bartels PH, Garcia FAR, Trimble CL, Kauderer J, Curtin J, Lim PC, et al. Karyometry in atypical endometrial hyperplasia: A Gynecologic Oncology Group study. Gynecol Oncol 2012; 125: $129-135$.

2. Glazer ES, Bartels PH, Prasad AR, Yozwiak ML, Bartels HG, Einspahr JG et al. Nuclear morphometry identifies a distinct aggressive cellular phenotype in cutaneous squamous cell carcinoma. Cancer Prev Res 2011; 4: 1770-1777.

3. Bartels PH, Bartels HG, Alberts DS, Yozwiak M, Prasad AR, Glazer ES, et al. Karyometry of nuclear phenotypes in cutaneous squamous cell cancer. Anal Quant Cytol Histol 2012; 34: 1-8.

4. Haass NK, Smalley KS. Melanoma biomarkers current status and utility in diagnosis, prognosis and response to therapy. Mol Diagn Ther 2009; 13: 283-296.

5. Sekula-Gibbs SA, Shearer MA. Sentinel node biopsy should be offered in thin melanomas with mitotic rate greater than one. Dermatol Surg 2011; 37: 1080-1088.

6. Bleicher RJ, Essner R, Foshag LJ, Wanek LA, Morton DL. Role of sentinel lymphadenectomy in thin invasive cutaneous melanomas. J Clin Oncol 2003; 21: 1326-1331.

7. Zukauskaite R, Schmidt H, Asmussen JT, Hansen O, Bastholt L. Asymptomatic brain metastases in patients with cutaneous metastatic malignant melanoma. Melanoma Res 2013; 23: $21-26$.

8. Bjoernhagen V, Erhardt K, Lagerloef B, Auer G. Comparison of nuclear DNA content in primary and metastatic malignant melanoma. Anal Quant Cytol Histol 1991; 5: 343-350.

9. Tan GJ, Baak JP. Evaluation of prognostic characteristics of stage I cutaneous malignant melanoma. Anal Quant Cytol Histol 1984; 6: 147-154.

10. Baak JP, Tan GJ. The adjuvent prognostic value of nuclear morphometry in stage I malignant melanoma of the skin. A multivariate analysis. Anal Quant Cytol Histol 1986; 8: 241-244.

11. Li LX, Krotty KA, Palmer AA, Kril JJ, Stankovic R, Skolyer R A, et al. Differentiating benign nevi from malignant melanoma using DNA microdensitometry and karyometry and maturation. Anal Quant Cytol Histol 2002; 24: 234-243.

12. Kruskal WH, Wallis WA. Use of ranks on one criterion variance analysis. J Amer Stat Assoc 1952; 47: 583-621. Addendum 1953; 48: 907-911.

13. Genchi H, Mori K. Evaluation and feature extraction on automatic pattern recognition system. Denki Tsuchin Gakkai Pari (in Japanese), 1965: 1.

14. Bartels PH, Olson GB. Computer analysis of lymphocyte images. In: Catsimpoolas N, ed. Methods of Cell separation, New York: Plenum Press, 1980: 1-99. 
15. Bartels PH, Bartels HG. Classification in karyometry, performance testing and prediction error. Anal Quant Cytol Histol 2013; 35: 181-188.

16. Davis PG, Serpell JW, Kelly JW, Paul E. Axillary lymph node dissection for malignant melanoma. ANZ J Surg 2011; 81: 462-466.

17. Neves R, Reynolds B, Hazard S, Saunders B,Mackay DR. Increased post-operative complications with methylene blue versus lymphazurin in sentinel lymph node biopsies for skin cancers. J Surg Oncol 2011; 103: 421-425.

18. Ul-Mulk J,Holmich LR. Lymph node dissection in patients with malignant melanoma is associated with high risk of morbidity. Dan Med J 2012; 59: A4441.

19. Roulin D, Matter M, Bady P, Lienard D, Gugerli O, Boubaker A et al. Prognostic value of sentinel node biopsy in 327 prospective melanoma patients from a single institution. Eur J Surg Oncol 2008; 34: 673-679.

20. Tsunoda K, Oikawa H, Tada H, Tatemichi Y, Muraoka S, Miura S, et al. Nucleus accumbensassociated 1 contributes to cortactin deacetylation and augments the migration of melanoma cells. J Invest Dermatol 2011; 131: 1710-1719.

21. Richards HW,Medrano EE. Epigenetic marks in melanoma. Pigment Cell Melanoma Res 2009; 22: 14-29.

22. Nagarajan RP, Costello JF. Epigenetic mechanisms in glioblastoma multiforme. Semin Cancer Biol 2009; 119: 188-197.

23. Lai CY, Schwartz BE, Hsu MY. CD 133+ melanoma subpopulations contribute to perivascular niche morphogenesis and tumorigenicity through vasculogenic mimicry. Cancer Res 2012; 72: 5111-5118.

24. Luo Y, Ellis LZ, Dallaglio K, Takeda M, Robinson WA, Robinson SE, et al. Side population cells from human melanoma tumors reveal diverse mechanisms for chemoresistance. $J$ Invest Dermatol 2012; 132: 2440-2450.

25. Setia N, Abbas O, Sousa Y, Garb JL, Mahalingam M. Profiling of ABC transporters ABCB5, ABCF2 and nestin-positive stem cells in nevi, in situ and invasive melanoma. Mod Pathol 2012; 25: 1169-1175. 
Figure 1. A bivariate plot of total optical density and relative nuclear area

This plot shows that the distribution of cases with potential progression and for cases that remain indolent cover the same range, and the plot also shows that progression to metastatic disease occurs independently of total optical density, i.e., even in cases with near diploid DNA content. 
Figure 2. Discriminant function scores of nuclei from both metastatic and non-metastatic melanoma lesions 
Figure 3. A histogram describing the relative proportion of aggressive nuclei in non-metastatic (top) and metastatic (bottom) melanoma cases 
Table 1. Mean values and standardized coefficients for features in the discriminant function and training set

$\begin{array}{llll}\text { Feature } & \text { M } & \text { Mmeta } & \text { coefficient } \\ \text { Discriminant Function } & & & \\ \text { Total optical density } & 0.628 & 0.593 & -0.043 \\ \text { Pixel O.D. histogram 0.9-1.0 } & 7.451 & 3.39 & -0.185 \\ \text { Avg. pixel O.D. } & 65.67 & 57.53 & -0.682 \\ \text { Cooccurr. Element 4-1 } & 7.94 & 6.33 & 1.299 \\ \text { Run length non-uniformity } & 7.45 & 3.48 & 0.54\end{array}$

Training Set

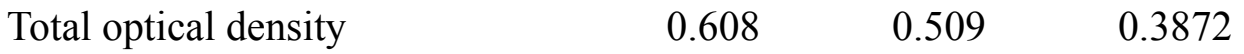

Pixel O.D. histogram 0.9-1.0 $\quad 9.58 \quad 6.1 \quad-0.4026$

$\begin{array}{llll}\text { Avg. pixel O.D. } & 69 & 58.4 & 0.6199\end{array}$

$\begin{array}{llll}\text { Cooccurr. Element 4-1 } & 8.44 & 4.5 & 0.5773\end{array}$ 
Table 2. Classification matrix for nuclei ${ }^{1}$

$\begin{array}{lcc} & \text { M } & \text { Mmeta } \\ \text { M } & 57.7 \% & 42.3 \% \\ \text { Mmeta } & 23.7 \% & 76.3 \%\end{array}$

${ }^{1}$ Overall, the average accuracy was $64.3 \%$. 
Table 3. The percent of nuclei in each data set assigned to aggressive/metastatic (Mmeta) group and the non-aggressive/non-metastatic $(\mathrm{M})$ group

$\begin{array}{cc}\text { M (\%) } & \text { Mmeta(\%) } \\ 82.1 & 54.5 \\ 25.0 & 77.5 \\ 30.0 & 41.2 \\ 60.6 & 83.3 \\ 1.7 & 27.7 \\ 54.0 & 71.3 \\ 10.3 & 51.0 \\ 58.9 & 100.0 \\ 45.0 & 100.0\end{array}$

51.2

24.4

15.1

6.2

42.4

10.9

38.2

24.3

$\begin{array}{ll}\text { Mean } \quad 33.95 \% & 68.5 \%\end{array}$ 